\title{
Análise da Não Participação Feminina nos Cursos de Informática em Escolas Estaduais de Educação Profissional
}

\author{
Sara Elen R. Sousa ${ }^{1}$, Katyeudo Karlos de S. Oliveira ${ }^{2}$, Carlos Williamy L. \\ Andrade $^{2}$, Eduarda P. de Sousa ${ }^{1}$, José Diener Feitosa M. Segundo ${ }^{1}$ \\ ${ }^{1}$ Centro Universitário Vale do Salgado (UniVS) \\ Av. Monsenhor Frota, 609 - São José, CEP 63430-000, Icó - CE - Brasil \\ 2Programa de Pós-Graduação em Informática Aplicada(PPGIA) \\ Universidade Federal Rural de Pernambuco(UFRPE)
}

Rua Dom Manoel de Medeiros, s/n, Dois Irmãos, CEP 52171-900, Recife - PEBrasil. \{sarethipetrova, karlos.1914. so, carloswilliamyl ourenco\} @gmail.com,
\{eduardasousa, josediener\}@fvs.edu.br

\begin{abstract}
It is known that gender inequality is still present in society, in all areas, including education. Areas such as technology are stereotyped as masculine. According to UNESCO, education must be inclusive and equitable. With this in mind, this article brings a study in order to understand the non-involvement of women in the Computer Science course in State Schools of Professional Education. A questionnaire was applied in order to contribute to the UN narrative, through a quantitative survey with 109 participants from 4 schools in 3 cities. It is concluded that, it is necessary to better explore the female presence in Informatics and establish actions to make it possible to achieve gender equality in education.
\end{abstract}

Resumo. Sabe-se que a desigualdade de gênero é algo ainda presente na sociedade, em todos os âmbitos, inclusive no educacional. Áreas como a tecnologia, são estereotipadas como masculinas. De acordo com a UNESCO, a educação deve ser inclusiva e equitativa. Pensando nisso, o presente artigo traz um estudo a fim de entender o não envolvimento de mulheres no curso de Informática em Escolas Estaduais de Educação Profissional. Foi aplicado um questionário com o objetivo de contribuir com a narrativa da ONU, a través de uma pesquisa quantitativa com 109 participantes de 4 escolas em 3 cidades. Conclui-se que, é necessário explorar melhor a presença feminina na Informática e estabelecer ações para que torne possível conquistar a igualdade de gênero na educação.

\section{Introdução}

O compromisso pela redução das desigualdades de gênero é um dos itens abordados pela Organização das Nações Unidas (ONU), presente nos Objetivos de Desenvolvimento Sustentável (ODS) - Agenda 2030. Em sua estrutura, o ODS 5 possui o intuito de alcançar a igualdade de gênero e empoderar todas as mulheres e meninas. Na meta 5.b, observa-se a finalidade de elevar o uso de tecnologias de base, 
em particular, as tecnologias de informação e comunicação para promover o empoderamento das mulheres [ONU 2016]. Porém, segundo a Organização das Nações Unidas para a Educação, a Ciência e a Cultura (UNESCO), o ODS 5 está intrinsecamente relacionado ao ODS 4, que visa garantir o direito à educação de qualidade, inclusiva e equitativa, com possibilidades de aprendizagem no decorrer da vida [UNESCO 2020].

Porém, no âmbito educacional, é perceptível a desigualdade entre gêneros. Segundo Freitas (2018), este tema é comumente manifestado nas instituições de ensino, podendo ser visto através dos problemas enfrentados pelas mulheres que escolhem ingressar e progredir na carreira acadêmica, onde os homens têm maior presença. Atualmente, áreas como a tecnologia são estereotipadas como masculinas, o que torna mais difícil a inclusão digital de mulheres, sendo necessário superar essas barreiras, procurando que, além de usuárias, as mulheres tornem-se participantes deste meio [Natansohn 2013]. O preconceito de gênero, ainda ocorre no sistema educacional em certos contextos e se mantém ao invés de ser rebatido [UNESCO 2020].

Globalmente, até 2017, as mulheres estavam presentes em somente 35\% no Ensino Superior em ciências, tecnologia, engenharia e matemática (STEM) [UNESCO 2018]. No Brasil, segundo dados da Sociedade Brasileira de Computação (SBC), a té o mesmo período, somente $14 \%$ dos matriculados na Computação eram do sexo feminino [Nunes 2018]. Tal fenômeno, acontece também nas escolas direcionadas à educação de nível técnico. Conforme Alves (2016), cursos técnicos que necessitam de um maior entendimento de tecnologias e engenharias, se tornam uma das causas que levam a exclusão do público feminino, pelo fator da estereotipização da área de informática. Da mesma maneira que acontece nos cursos de Graduação e Pós-Graduação, a presença feminina em profissões de nível técnico é desproporcional a outras áreas, fazendo com que as mulheres sejam mais presentes em alguns setores como, tarefas pessoais, educação e saúde.

Destarte, o objetivo deste artigo consiste em analisar os possíveis motiv os que fazem com que as mulheres não entrem no curso de Informática em Escolas Esta duais de Educação Profissional.

Para tanto, além deste tópico introdutório, este trabalho está organizado da seguinte forma: a Seção 2 descreve os materiais e métodos que orientaram a pesquisa; a Seção 3, traz os trabalhos relacionados; na Seção 4, é apresentada a análise dos dados; e, por fim, a Seção 5 aborda as considerações finais e trabalhos futuros.

\section{Materiais e Métodos}

Este trabalho, está metodologicamente ancorado em uma pesquisa exploratória de natureza quantitativa, com procedimentos de estudo bibliográfico [Gil 2002]. Objetivando avaliar aspectos envolvendo possíveis preconceitos, discriminações e o não envolvimento das mulheres na tecnologia, foi utilizado como instrumento de coleta de dados, um questionário aplicado por meio do Google Forms (aplicativo de formulários disponibilizado pela Google).

O questionário foi formado por 4 perguntas objetivas, que abordavam o conhecimento da existência do curso de Informática na instituição, motivo pelo qual as meninas não escolheram este curso, a relevância da Informática para a sociedade e a 
importância da participação feminina neste meio. O estudo foi realizado com um grupo de 109 alunas de 4 escolas profissionalizantes de ensino médio da rede pública de 3 cidades. Como critério de exclusão, não pôde ser aplicado com alunas do curso de Informática, pois, o intuito da pesquisa era analisar os eventuais motivos que fizeram elas não entrarem no citado curso. A pesquisa foi realizada entre o decorrer dos meses de janeiro e fevereiro do ano de 2020.

\section{Trabalhos Relacionados}

Em um estudo realizado por Marinho, Fagundes e Aguilar (2019), foi investigado a participação feminina em cursos técnicos e de graduação em Informática. A princ ípio, as autoras afirmam que um dos fatores para as mulheres não ingressarem na área, é o machismo enraizado na educação de meninas, como também, o fato de que houveram mulheres que foram importantes para a tecnologia, mas que não foram, ou não são reconhecidas. De acordo com dados levantados pelo estudo, é possível perceber a discrepância entre mulheres e homens nos cursos analisados, sendo nítida a diferença do percentual obtido na análise dos dados. O estudo mostra também, que a maioria das mulheres que ingressam nos cursos, acabam abandonando-o no meio da jornada.

Assim, conclui-se no trabalho, que é necessário existir práticas inclusivas no âmbito da tecnologia pois, segundo as autoras, um grupo com maior diversidade em etnia, gênero e sexo, tende a ser mais inovador e criativo. Ainda, é visto que na área de tecnologia da informação, é necessário e importante existir o trabalho em equipe, sendo ainda mais interessante que haja diversidade, porém, a presença feminina no meio é ainda retraída e em pouca quantidade.

Em outro estudo, realizado por Sarmento (2018), é analisado a participação e desempenho dos gêneros no Centro de Informática da UFPB (Universidade Federal da Paraíba). O estudo mostra que essa diferença está presente também no mercado de trabalho, onde as mulheres têm os salários inferiores ao dos homens, assim como nas universidades. O trabalho traz diversos dados de discriminação de gênero, principalmente, na área da computação a nível mundial. Observa que a discrepância de gênero é real dentro do ambiente analisado, e evidencia a importância da temática para o desenvolvimento de projetos no incentivo da participação feminina em cursos da área. Concluindo também que, é importante que haja projetos que trabalhem o incentivo do ingresso de mulheres na área.

No trabalho realizado por Soares (2018), a autora retrata a discriminação contra mulheres técnicas em manutenção de computadores no mercado de trabalho. A autora fez uma coleta de dados através de um questionário com alunos de diversos cursos do Instituto Federal de Educação, Ciência e Tecnologia do Rio Grande do Norte. No estudo, constatou-se que mais de $50 \%$ dos entrevistados não associam o sexo feminino à área, mostrando assim, a presença do problema. No decorrer do trabalho, a autora afirma que a figura feminina é de grande importância para o crescimento da tecnologia. Ademais, foi possível concluir que há preconceitos com o sexo feminino neste âmbito, o que faz a presença de mulheres se tornar cada vez menor, provocando um questionamento sobre a ausência do público feminino na área.

Uma questão fundamental que difere este trabalho dos demais, é a investiga ção dos possíveis motivos do não ingresso do público feminino na área de Informática. 
Outra questão importante, é que o estudo foi aplicado no ensino profissionalizante, e m Escolas Estaduais de Educação Profissional.

\section{Resultados e Discussão}

O questionário foi idealizado com o objetivo de contribuir com a narrativa da $\mathrm{ONU}$ (2016), que afirma que o debate sobre a distribuição de gênero mais uniforme, tornouse uma questão primordial para a entidade, visto que a desigualdade é um fato presente, tanto em nações desenvolvidas, quanto em subdesenvolvidas. Também, com a recente estratégia da UNESCO (2020), que guiará as ações da entidade para estruturar as concepções e ações transformadoras, para que seja possível conquistar a igualdade entre gêneros na educação e por intermédio da educação [UNESCO 2020].

Na primeira pergunta (Figura 1), foi questionado se a aluna tinha conhecimen to da existência do curso de Informática na escola em que ela estava estudando. Observase que $84,4 \%$ das participantes afirmaram ter conhecimento que a instituição de ensino ofertava o curso de Informática. Ainda, 15,6\% mostraram que não sabiam da existência do curso.

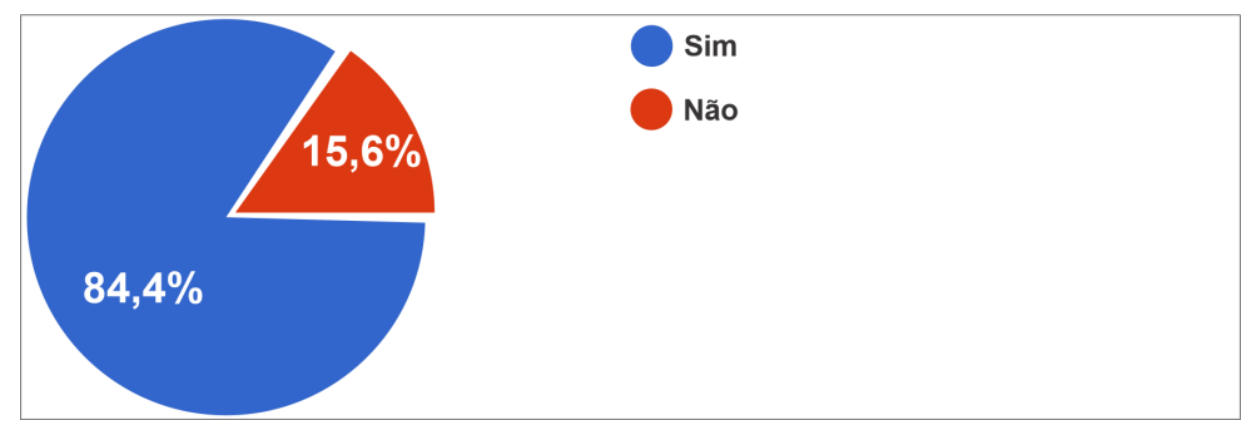

Figura 1. Conhecimento sobre a existência do curso de Informática na escola

Na segunda pergunta (Figura 2), foi questionado o motivo pelo qual a aluna não escolheu o curso de Informática da instituição. Percebe-se que, 51,4\% das participantes afirmam que o maior motivo para que não ingressassem no curso é "Nunca foi uma opção minha entrar nessa área". Nunes et al. (2015) dizem que, é perceptível o desinteresse das mulheres neste âmbito, tornando escassa a participação feminina na área.

Também, pode-se perceber que $16,5 \%$ tinham dúvida/medo por não conhecerem outras mulheres no curso ou trabalhando na área, assim, concordando com Marinho, Fagundes e Aguilar (2019), quando é mencionado outro fator significativo para a não participação das mulheres nos cursos de tecnologia, a falta de representatividade feminina na área. As autoras realçam que, diversas mulheres marcaram presença no desenvolvimento da história da computação, mas os homens geralmente são mais citados e, raramente, alguém que não for da área tecnológica, terá conhecimento dos feitos realizados por essas mulheres. 


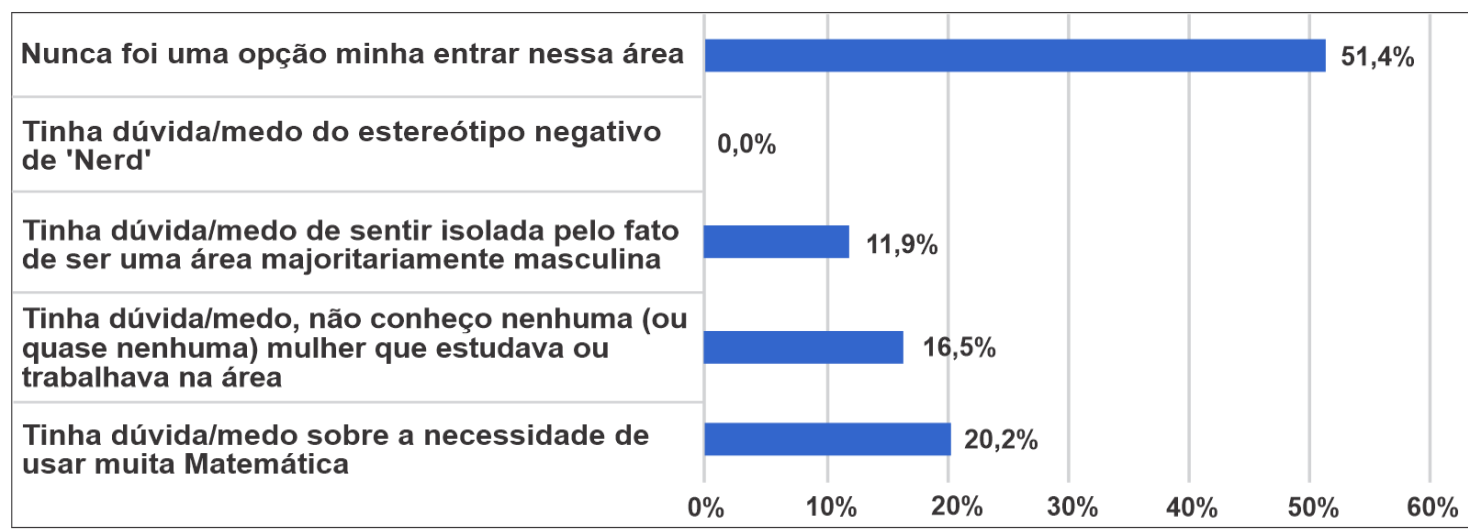

Figura 2. Motivo de não escolher o curso de Informática

Nessa perspectiva, conforme a UNESCO (2018), as mulheres estão mais presentes de forma predominante em campos vistos como femininos, por exemplo, educação e saúde, e os homens permanecem como maioria em cursos de tecnologia da informação e comunicação e as engenharias. Nos cursos de Informática, estudos apontam as mulheres como minoria em todo o mundo, não sendo diferente no Brasil [Monard e Fortes 2013, Ashcraft, McLain e Eger 2016].

Em seguida, a coleta nesta parte do questionário se dá por uma escala de tipo Likert de 5 pontos, com valores entre "concordo totalmente" (5) até "discordo totalmente" (1). Aqui, as perguntas serão referidas através de um ID (identificador), conforme a Tabela 1 .

Tabela 1. Perguntas escala Likert

\begin{tabular}{|l|l|}
\hline ID & \multicolumn{1}{|c|}{ Pergunta } \\
\hline Q3 & $\begin{array}{l}\text { Você concorda que o curso de Informática é importante para o desenvolvimento da } \\
\text { sociedade? }\end{array}$ \\
\hline Q4 & Você concorda que é importante existir mais mulheres na área de tecnologia? \\
\hline
\end{tabular}

Observando o gráfico referente a "Q3" (Figura 3), observa-se que, o resultado obtido foi de $74,3 \%$ para "concordo totalmente" e $21,1 \%$ "concordo", que "sim", o curso de Informática é importante para o desenvolvimento da sociedade. Assim, é possível perceber uma contraposição nas respostas das participantes, se comparado ao gráfico 2 (Figura 2), onde as entrevistadas não têm a Informática como opção, ou seja, a área da Informática possui importância para a sociedade (na visão das entrevistadas), mas, tal fato possivelmente não foi levado em consideração na escolha do curso.

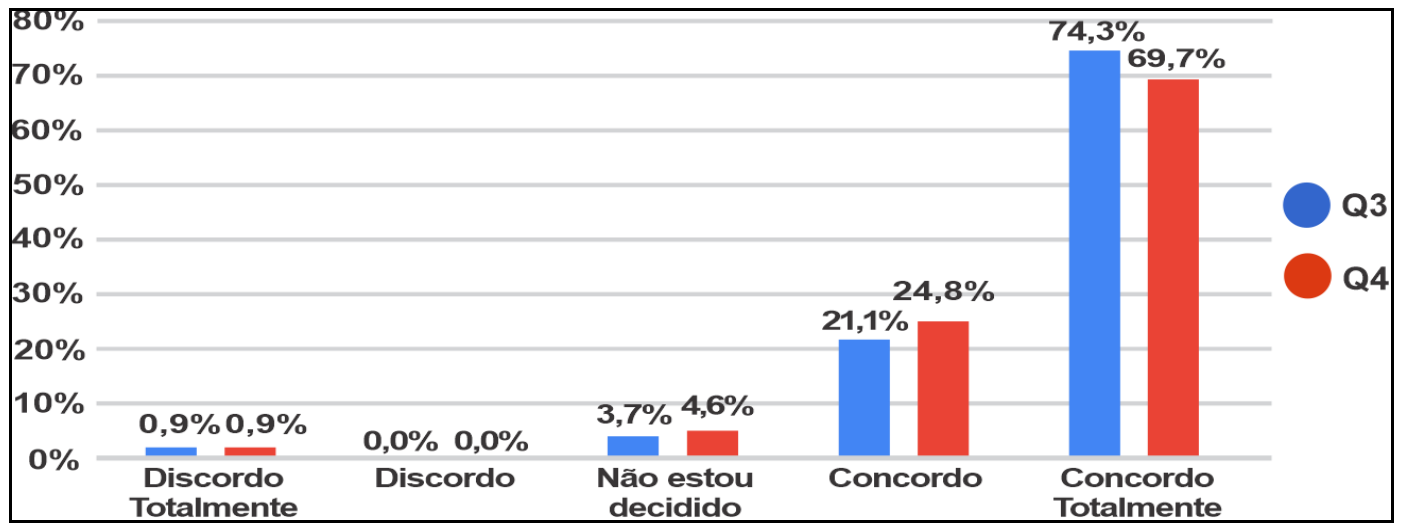

Figura 3. Importância do curso de Informática para o desenvolvimento da sociedade 
Na análise de "Q4" no gráfico (Figura 3), nota-se que 69,7\% diz que "concordo totalmente" e $24,8 \%$ "concordo", que "sim", seria algo positivo se houvessem mais mulheres na tecnologia. A partir disso, é importante lembrar o valor da participação feminina na área, Carvalho e Casagrande (2011), colocam em evidência nomes femininos importantes para o desenvolvimento da tecnologia e da ciência. Dito isto, é possível assegurar que o conhecimento das mulheres é extenso e capaz de ir muito mais além do que é preconceituosamente determinado pela sociedade. Ainda, conforme Flores (2013), seria positivo se existissem mais mulheres na tecnologia, vis to que em seu estudo foi evidenciado que is to causaria uma diversidade mais ampla, menos preconceito, maior igualdade e inovação na área

Apesar do papel essencial exercido por mulheres no decorrer da evolução da Computação, a desigualdade de gênero ainda é muito notória [Heinzmann et al. 2017]. Estereótipos associados a gênero, razões culturais e socioeconômicas, podem ser vistas como os principais motivadores pelo distanciamento de mulheres dos cursos da área de exatas [Cheryan, Master e Meltzoff 2015]. Conforme Scheckler (2008), estudos e iniciativas a nível institucional, são fundamentais para que seja possível aumentar o número de mulheres na computação, um meio que, de acordo com a autora, ainda resiste em admitir que existam problemas de gênero.

\section{Conclusões}

Este trabalho teve como objetivo, analisar o não ingresso das mulheres no curso de Informática em Escolas de Ensino Profissionalizante, através de uma pesquisa exploratória de natureza quantitativa, utilizando-se também, de um estudo bibliográfico.

Através da análise dos resultados, foi possível verificar que as entrevistadas conhecem o curso de Informática ofertado pela instituição de ensino, em sua maioria reconhecem como sendo de grande importância para o desenvolvimento da socie da de, porém, não escolhem o curso por não ser uma opção ou até mesmo por algum tipo de dúvida ou medo associado a diversos fatores. Ainda, as entrevistadas consideram que a presença de mulheres na informática é algo positivo e deveria existir em maior número. Assim, sendo analisando como possíveis causas para que aconteça a baixa representatividade do público feminino na área da tecnologia.

Ao avaliar o escopo e execução desta pesquisa, não foram constatadas ameaças à validade interna da análise dos resultados, todavia, menciona-se que os elementos experimentais foram designados a partir de poucas fontes (3 cidades, 4 escolas e 109 alunas), podendo possuir atributos intrínsecos que não existem em outros contextos. Dessa forma, podendo configurar uma possível ameaça à validade externa, no que diz respeito a escolha e tratamento da amostra, impedindo a generalização dos re sultados obtidos além do escopo analisado.

Assim sendo, encontra-se a necessidade de explorar de forma mais detalhada a presença feminina na Informática, como também, estabelecer ações para atrair e estimular a entrada do público feminino na área, assim como, preservar a continuidade das mulheres que já se encontram nos cursos de Informática. Ainda, tendo em vista a problemática abordada pelo estudo, e as várias discussões a respeito dos conceitos prédeterminados pela sociedade, espera-se que este trabalho contribua para um maior e melhor debate sobre questões de gênero na área analisada. 
Como trabalhos futuros, pretende-se ampliar o número de participantes do estudo, bem como, a quantidade de escolas. Ainda, será executada uma investigação qualitativa, com o intuito de analisar as questões de preconceito e discriminação no meio. Desta forma, será possível obter um perfil mais detalhado, elaborar uma estratégia de conscientização, divulgação e ações de debate sobre gênero na tecnologia, com o intuito de atrair mais meninas para a área. Também, investigar índices de aproveitamento, evasão e convívio social com alunas que fazem o curso de Informática, para que seja possível obter informações suficientes e, assim, implantar em Escolas Estaduais de Educação Profissional, projetos de extensão da Instituição de Ensino Superior dos autores deste trabalho.

\section{Referências}

Alves, H. V. S. (2016). "Educação profissional e percepção de gênero: uma investigação entre alunas e alunos do Serviço Nacional de Aprendizagem Comercial Senac de Porto Velho-RO”. Formação (Online), 4(23).

Ashcraft, C., McLain, B. and Eger, E. (2016). Women in tech: The facts. National Center for Women \& Technology (NCWIT).

Carvalho, M. G. and Casagrande, L. S. (2011). Mulheres e ciência: desafios e conquistas. INTERthesis: Revista Internacional Interdisciplinar, 8(2), 20-35.

Cheryan, S., Master, A., and Meltzoff, A. N. (2015). Cultural stereotypes as gatekeepers: increasing girls interest in computer science and engineering by diversifying stereotypes. Frontiers in Psychology, 6:49.

Flores, B. D. S. (2013). "Uma análise preliminar da participação feminina em cursos na área da Computação da Grande Porto Alegre". Trabalho de Conclusão de Curso (Graduação em Ciência da Computação). Universidade Federal do Rio Grande do Sul, Instituto de Informática, Porto Alegre.

Freitas, M. A. (2018). "Assimetrias de gênero na perspectiva de mulheres acadêmicas de uma universidade federal brasileira". Tese (Doutorado em Educação). Faculd ade de Educação, Universidade Federal de Minas Gerais, Minas Gerais.

Gil, A. C. (2002). Como elaborar projetos de pesquisa (Vol. 4, p. 175). São Paulo: Atlas.

Heinzmann, L., Romagna, M. E., Gosteinski, M. A. C. and Silva, J. M. C. (2016). Meninas e jovens na computação. In Anais do Computer on the Beach, pages 554558, Florianópolis, SC, Brasil.

Marinho, G., Fagundes, S. and Aguilar, C. (2019). Análise da participação feminina nos cursos técnicos e de graduação da área de Informática da Rede Federal de Educação Tecnológica e do Cefet/RJ campus Nova Friburgo. In Anais do XIII Women in Information Technology (pp. 21-30). SBC.

Monard, M. C. and Fortes, R. P. D. M. (2013). Uma Visão da Participação Feminina nos Cursos de Ciência da Computação no Brasil. In V Congresso de la Mujer Latinoamericana em La Computacion. Caracas.

Natansohn, G. (2013). "O que tem a ver as tecnologias digitais com o gênero?". feminino Internet em código, 15. 
Nunes, D. (2018). Educação superior em computação, estatísticas 2017. Sociedade Brasileira de Computação - SBC.

Nunes, M. M., Rodrigues, L. F., Martinhago, A., Soares, L. and Reis, R. (2015). Uma iniciativa para atrair as estudantes do ensino médio para a Área de Tecnologia da Informação. In Anais do Workshop de Informática na Escola (Vol. 21, No. 1, p. 425).

ONU. Nações Unidas Brasil. (2016) "Glossário de termos do Objetivo de Desenvolvimento Sustentável 5: Alcançar a igualdade de gênero e empoderar to das as mulheres e meninas", https://nacoesunidas.org/wpcontent/uploads/2017/06/Glossario-ODS-5.pdf, Janeiro.

Sarmento, R. A. V. (2018). "Plataforma para estudo de participação e desempenho dos gêneros no Centro de Informática". Trabalho de Conclusão de Curso (Graduação em Engenharia de Computação). Centro de Informática, Universidade Federal da Paraíba, João Pessoa.

Scheckler, R. K. (2008). Gender Myths and Beliefs in Computer Science. ABC-CLIO, Inc., Santa Barbara, California, 115-122.

Soares, A. G. N. (2018). "Avaliação da atuação das mulheres no mercado de trabalho na área de manutenção de computadores". Relatório de Conclusão de Curso (Técnico em Manutenção e Suporte em Informática). Instituto Federal de Educação, Ciência e Tecnologia do Rio Grande do Norte, Natal.

UNESCO (2018). Decifrar o código: educação de meninas e mulheres em ciências, tecnologia, engenharia e matemática (STEM). Brasília: Organização das Nações Unidas para a Educação, a Ciência e a Cultura.

UNESCO (2020). Do acesso ao empoderamento: Estratégia da UNESCO para a igualdade de gênero na e por meio da educação para 2019-2025. Paris: Organização das Nações Unidas para a Educação, a Ciência e a Cultura. 